\title{
Modeling the Impact of UAVs in Sustainability
}

\author{
José M. Conejero \\ Quercus Software Engineering Group \\ Universidad de Extremadura \\ 10004, Spain \\ Email: chemacm@unex.es
}

\author{
Isabel Sofia Brito \\ Instituto Politécnico de Beja \\ Campus do Instituto Politécnico \\ 7800-295 Beja, Portugal \\ Email: isabel.sofia@ipbeja.pt
}

\author{
Ana Moreira, Jácome Cunha \\ and João Araújo \\ NOVA LINCS, Universidade NOVA de Lisboa \\ 2829-516 Caparica, Portugal \\ Email: \{amm,jacome,joao.araujo\}@fct.unl.pt
}

\begin{abstract}
In the last few years, sustainability has become one of the priority lines for many companies and organizations, especially public administrations. This trend has been even more evident in some regions where the preservation of natural resources is of utmost importance, not only from an environmental perspective, but also from an economic one. In this context, technology has become one of the key factors to achieve sustainability goals. An example of these technologies are Unmanned Aerial Vehicles (UAVs) which are being used more and more with sustainability purposes. However, although some efforts have been made to propose software approaches to model sustainability, some examples that model the impact of technology on sustainability are still needed. This paper presents an instance of a sustainability metamodel for the UAVs domain. This model allows to specify the impact of UAV-based processes on sustainability, and also to identify potential limitations that may hinder its applicability. Finally, the paper provides some suggestions to complete the metamodel based on the instantiation process.
\end{abstract}

\section{INTRODUCTION}

Sustainability has become one of the priority lines of many public administrations and governments roadmaps. Indeed, sustainability goals are present in many political campaigns, governmental programs and global meetings. Such interest on sustainability spreads across countries and at a national level is being promoted with dedicated funds for innovation and development in a wide range of areas. For example, in regions where the main economic resource comes from cattle raising, agriculture and forest protection, special programs are being launched to foster sustainability through a better use of resources. A frequent common factor in these programs is the use of new technologies to achieve the goals proposed.

An example of these new technologies is the utilization of UAVs (Unmanned Aerial Vehicles, also known as drones). UAVs are being used in more and more contexts and domains due to the wide range of direct usages they provide. Examples of these application domains are security, sports, publicity, tourism or military defence. More recently, UAVs are also highly and successfully used to support environmental sustainability, such as cattle raising ${ }^{1}$, precision agriculture [1], control of PV plants [2], forests protection against fires [3], [4], [5], and oceans exploration [6]. Another interest area of application is social aspects related to

${ }^{1}$ Locating cattle with unmanned aerial vehicles, http://www.csiro.au/ en/Research/AF/Areas/Animal-Science/Precision-livestock-management/ Locating-cattle sustainability, such as rescue in catastrophes [7] or medical support $^{2}$. UAVs provide important benefits in these domains mainly due to their energetic efficiency, their reduced carbon footprint and their decreasing cost, when compared to the alternatives more commonly used [8], [9]. This is why many of the aforementioned organizations and administrations are considering the use of these small vehicles to modernize some traditional services they provide or even offering new and innovative ones that may help to achieve important sustainability goals ${ }^{345}$.

The incorporation of this new technology in those processes, requires approaches able to specify and quantify (by means of indicators and metrics) the real benefits. In the last few years there has been a trend in the software engineering community to consider sustainability as a first class citizen in the development of software. The result is the number of approaches that have emerged to model the concepts related to sustainability [10], [11], [12]. Most of the modeling approaches focus on the specification of sustainability-related concepts at the very beginning of the development process, in requirements engineering stages [13]. However, advances in techniques to measure the energy spent in running software, identifying the more energy-consuming pieces and apply refactorings to substitute those pieces with more energy efficient ones is also a current trend in research [14], [15], [16].

This paper takes one of these particular approaches to model sustainability and specifies the benefits, and drawbacks, of using UAVs to support some environmental related issues addressed by organizations, particularly public administrations. To this purpose, we use the metamodel introduced in [10] to build a model that details how activities performed with UAVs may impact on the sustainability of the environment. Moreover, the instantiation of this metamodel is also helpful for the evaluation of its utility. Hence, the contributions of this paper are the next:

1) present a new instantiation of the aforementioned

\footnotetext{
${ }^{2}$ Google Patent: http://goo.gl/NmXc5F

${ }^{3}$ Tanzania anti-poaching initiative, http://africageographic.com/blog/ tanzania-use-drones-new-anti-poaching-initiative/

${ }^{4}$ Extremadura Drone Territory Event, http://cicytex.gobex.es/es/eventos/51/ territorio-dron-extremadura

${ }^{5}$ Civil UAVs initiative in Galicia, http://gain.xunta.es/artigos/466/civil+ uavs+initiative
} 
metamodel in a domain where it had not yet been applied;

2) provide suggestions to improve the metamodel according to the experience of generating that new instantiation;

3) offer a model describing the benefits of using UAVs, clearly specifying their limitations (e.g., those due to the strict aerial regulations, that could hinder their use), that can be used by managers to support decisions related with adapting some of their organizational processes.

The rest of this paper is organized as follows. Section 2 presents the background needed to make the paper self-contained. Section 3 introduces a running example that will be used to motivate the work. Section 4 presents the new instantiation of the sustainability metamodel for the UAVs domain. Finally, section 5 and 6 presents related work and concludes the paper, respectively.

\section{BACKGROUND}

This section provides an introduction to the main topics involved in this work. It starts with an introduction to sustainability in software engineering (sub-section 2.1), then offers an overview of the metamodel that will be instantiated in this work (sub-section 2.2) and, finally, briefly introduces UAVs and their supporting technology (sub-section 2.3).

\section{A. Sustainability in Software Engineering}

In the few last years, the software engineering community has made an important effort to introduce sustainability as a primary focus on software development [17], [18], [19], [20]. Therefore, it is not surprising that several definitions of sustainability have been recently provided, some of them collected in [21], where for example, software sustainability is defined as a composite, non-functional requirement which is a measure of a system's extensibility, interoperability, maintainability, portability, reusability, scalability, and usability [21]. The Software Sustainability Institute claims that sustainability means that the software you use today will be available - and continue to be improved and supported - in the future [21]. Naumann et al. distinguish between sustainable software and sustainable development. While sustainable software is defined as software, whose direct and indirect negative impacts on economy, society, human beings, and environment that result from development, deployment, and usage of the software are minimal and/or which has a positive effect on sustainable development, sustainable software development is defined as the art of developing sustainable software with a sustainable software engineering process so that negative and positive impacts result in and/or are expected to result from the software product over its whole life cycle are continuously assessed, documented, and used for further optimization of the software [22].

There are several categorizations of sustainability. The United Nations defined a set of ten themes ranging from economic to social aspects of sustainability [23]. Goodlan also provided a categorization for general sustainability based on four different dimensions: individual, social, economic and environment [24]. The latter three were also defined as themes by the United Nations. However, as it was claimed in [10], an additional category related to technology is needed in order to consider sustainability in software systems so that they added one dimension to those considered by Goodlan. In this work, we also consider these five dimensions, defined as follows:

- Individual sustainability. Refers to private goods and individual human capital.

- Social sustainability. Relates to societal communities (mainly based on solidarity).

- Economic sustainability. Refers to assets, capital and, in general, added value achieved by the improvement of sustainability in a particular context.

- Environmental sustainability. Includes those activities performed to improve human welfare by protecting natural resources.

- Technical sustainability. Relates to the long-time usage of software systems and their adequate evolution over time.

\section{B. Penzenstadler and Femmer's metamodel}

In [10], the authors introduced a metamodel for sustainability that allows its instantiation for specific company processes or products. Fig. 1 depicts this metamodel, which is based on the definition of six different entities representing the main concepts involved in the specification of sustainability for specific processes. The metaclasses modelled are the following:

- Goal: represents the main objective that an instantiated model would be defined for. A goal may be composed by different related dimensions. In our case, the main goal will be "Sustainability".

- Dimension: represents the different aspects or viewpoints related to sustainability. The authors consider the five dimensions mentioned in previous section. A dimension influences other dimensions (sometimes in a conflicting way) and usually has a set of values related.

- Value: represents an intention related to the dimension being defined. This intention is usually perceived as a goal to be accomplished that may contribute to that dimension. An example of value could be "Reduce resource consumption by $30 \%$ within 12 months". A value may also have subvalues.

- Indicator: denotes a qualitative or quantitative metric to express the degree of fulfillment of a particular value. In other words, indicators approximate the value that is related to. Examples of these indicators are satisfaction indexes or carbon emissions.

- Regulation: denotes legal issues or restrictions that may affect the processes defined by the model. Examples include the emission regulations established by the European Union. These regulations support the achievement of the values and also influence the indicators.

- Activity: represents an action performed to contribute to a value related to a dimension and, thus, influences on 


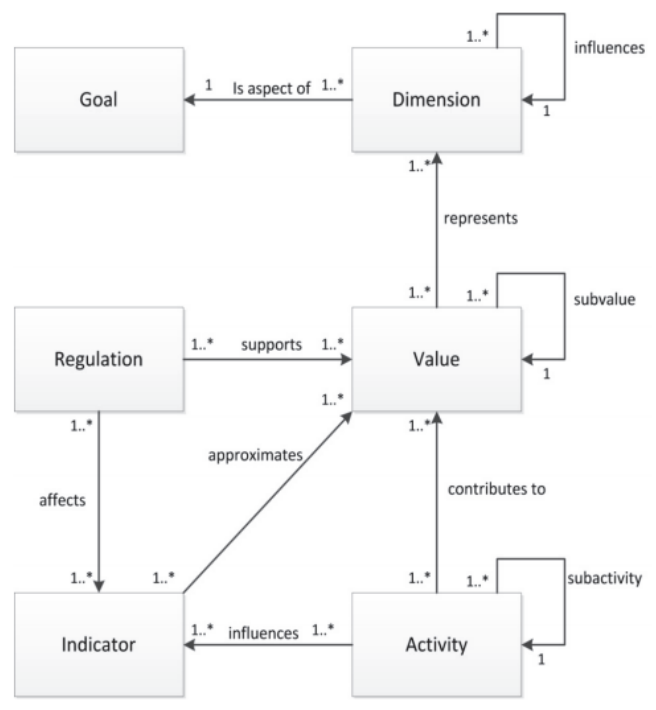

Fig. 1. Metamodel for sustainability presented in [10]

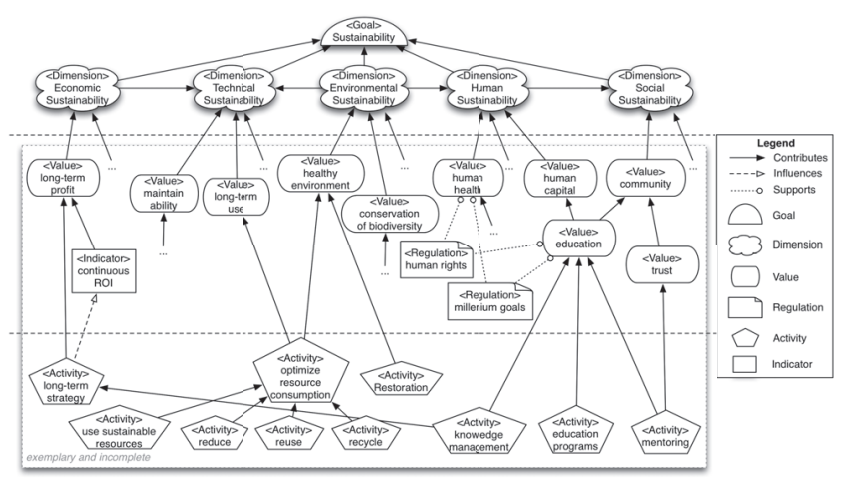

Fig. 2. Generic model for sustainability presented in [10]

the indicators. An activity may be composed of other subactivities. A concrete example could be "Establish incentives for less resource consumption".

Based on this metamodel, the authors instantiated a generic model that may be reused when considering sustainability in different contexts (both company and product-specific domains). This model is illustrated in Fig. 2. This model contains the five aforementioned dimensions related to sustainability and different values and activities that may recurrently appear in instantiations for several domains.

\section{C. $U A V S$}

According to the UK UAVs Association, UAVs may be defined as a reusable aerial vehicle that is capable of operating without an internal pilot, is tethered by a radio control link, and can be preprogrammed for both flight and payload operations prior to launch [25]. There are several types of UAVs each one with many different purposes and different sizes, weights or hardware configurations. However, one of the types that have contributed more to the wide extension of this technology is the multicopter, characterized by being able to take off and on from a static position.

From a technological perspective, this type of UAVs are usually composed by the following set of hardware and software components [26]:

- RC Transmitter: this component allows the pilot to remotely control the device. This component may not be required for some UAVs that perform autonomous flights.

- Multi-rotor frame: this refers to the hardware chassis that is used to support the placement of the rest of components and connect them. It also contains the arms for the airscrew and motors. The number of arms may range from three (tricopter) to eight (octocopter) or more.

- Motors/Speed controller: motors highly influence the device's weight. Usually they should be all of the same type in order to supply the same power. However, they may provide different speeds to the airscrews that are controlled by the speed controller.

- Flight controller/AutoPilot: this component is responsible for controlling each motor so that the device may be stabilized in a flight. It is also known as the AutoPilot and it could be considered the device's brain.

\section{A REAl CASE: EXTREMAdura AgroTech}

This section presents a motivating case that will drive the explanation of the model instantiations presented in this work. This case is derived from a real project proposed in the region of Extremadura, in Spain. Extremadura is one of the regions with lower industrialization degree in the country. By contrast, its economical model is mainly based on the exploitation of natural resources, namely cattle industry, agriculture and rural tourism. The government of the region developed a strategy that encourages the use of new technologies not only to improve the performance of these natural resources, but also to preserve them ${ }^{6}$. This project was called Extremadura AgroTech ${ }^{7}$ and one of its priority lines was the use of UAVs for sustainability purposes.

To foster this priority line, the administration launched an open call for ideas and projects for using UAVs in areas such as (we focus on a limited set of them for the sake of brevity):

- Early detection of fires;

- Early detection of plagues;

- Distribution of pesticides;

- Detection of robberies at fields;

- Measurement of different kind of environmental indexes (e.g., pollution, pollen, acoustic, illumination);

- Animal behaviour control (e.g., bird routes, births);

- Water irrigation decisions.

Note that some of these projects would, obviously, provide important benefits for sustainability in the region. However,

\footnotetext{
${ }^{6}$ Research and Innovation Strategy for Specialization in Extremadura, http://one.gobex.es/docs/Estrategia_RIS3_Extremadura.pdf

${ }^{7}$ FabLab AgroTech Extremadura, http://fablab.cenatic.es/
} 
how could these benefits be measured? What would be the impact of deploying all these projects on sustainability? Next section shows how the use of modeling techniques could help to specify not only this impact but also the potential limitations of the solutions. Note that, for instance, these devices are strongly limited by the strict aerial regulations that they must fulfil so that an analysis of these applications should be performed to ensure their utilization.

\section{A MODEL FOR SUSTAINABILITY USING UAVS}

This section presents a requirements model used to specify the relation between the UAVs applications presented in previous section and sustainability. The model has been built as a new instantiation of the metamodel introduced in Section 2.2. In particular, as the authors describe in [10], this metamodel allows building not only product-specific instances but also company or organization-specific ones. In our case, the instantiation presented in this work belongs to the latter category since it models the sustainability policy for a big organization interested in introducing UAVs in their processes.

As it is explained in [10], the instantiation of the metamodel for companies consists of two different steps: i) the analysis phase and ii) the application and assessment phase. While the former refers to the process of making concrete the definition of sustainability in the company context, the latter implies the execution of the selected activities and their assessment in terms of checking whether they are working. In this paper we mainly focus on the first activity as a key step to achieve the second one.

To perform the analysis phase, a goal oriented model has been built where the generic goal model presented in [10] has been tailored to the particular context of UAVs. To this purpose, we followed the substeps proposed by the authors: 1) for each dimension, we instantiated the generic values, paying special attention to potential conflicts among them (e.g., contradictory goals); 2) activities to implement those goals are proposed together with indicators that allow approximating the achievement for each goal; 3) once activities and indicators are defined, the influence of the activities on the indicators is analyzed to determine their positive or negative impact.

Note that the $<$ Dimension $>$ metaclass included in the metamodel allows specifying the five dimensions of sustainability previously mentioned. The following subsections present the environmental, economic, technical and social model dimensions. The individual model dimensions is not presented for the sake of brevity.

\section{A. Environmental sustainability supported by UAVs}

Fig. 3 shows an excerpt of the model built for the environmental dimension. The model has been built in three layers [10]: dimensions (top layer), values, indicators and regulations (middle layer), and activities (lower layer). Moreover, since the authors also provided a generic instantiation of the metamodel, some of the entities included in this instantiation could be reused in the instantiation for other products or companies. For example, the $<$ Value $>$ entities "Reduce energy consumption" and "Conservation of biodiversity" have been reused from the generic instance introduced in [10]. Similarly, some of the new entities included in the model presented here could be easily reused in other processes. It is worth mention that, although not explicitly mentioned in the name of the $<$ Activity $>$ entities defined in our model, those activities are all related to UAVs so that they would be performed by using these devices.

Based on the model shown in Fig. 3, the managers of the organization may observe the impact of using drones in the traditional (or new) processes that the organization performs. For instance, the "Fly region with thermal cameras" activity will be carried out by drones equipped with those kind of cameras that will perform programmed flights in particular regions of the forests where fires represent a potential risk. The early detection of these fires would reduce the areas of burnt forests so that this activity contributes to the achievement of the "Reduce fire areas" value. Moreover, since the traditional mechanisms that the organization uses to this purpose are based on supervising these areas by $4 \times 4$ vehicles, the reduction in the energy consumed to perform this task will be also considerable, since the used UAVs may be supported just by batteries. This is why that activity influences positively on the "Energy bill" indicator. The rest of activities also influence (positively or negatively) in this indicator since, on the one hand, the use of UAVs may imply a reduction of energy consumption when they are used to replace traditional vehicles; and, on the other hand, they may lead to an extra energy consumption when they support new processes. Similarly, the "Detect dry zones and humidity" activity supports the indicator "Water consumption" since the irrigation of fields may be dynamically adapted to the climatic conditions. The "Energy bill" and "Water consumption" indicators contributes to achieve the "Reduce energy consumption" and "Reduce natural resources consumption" values, respectively.

The model also shows some regulations that may hinder the achievement of some of the goals. We used the $<$ Regulation $>$ metaclass to represent these limitations. As an example, observe that the "Reduce pollution" value is supported by three different regulations. Since detecting pollution may include flights over populated areas (to measure some pollution indexes), this value is restricted by the requirements that a drone must fulfil to fly over a populated area (according to the local regulations for our motivating case). By contrast, the activities related to flights over forest or sown field may be autonomously carried out (being previously programmed). Thus, these activities are restricted by the "Avoid populated area" regulation, otherwise, these flights would require a pilot.

\section{B. Economic sustainability supported by UAVs}

Part of the model for the economic dimension is presented in Fig. 4. This model shows the impact of using drones in the processes but, in this case, related to financial issues. Note that some entities may appear in several dimensions since, for instance, they may influence the environment but also provide 


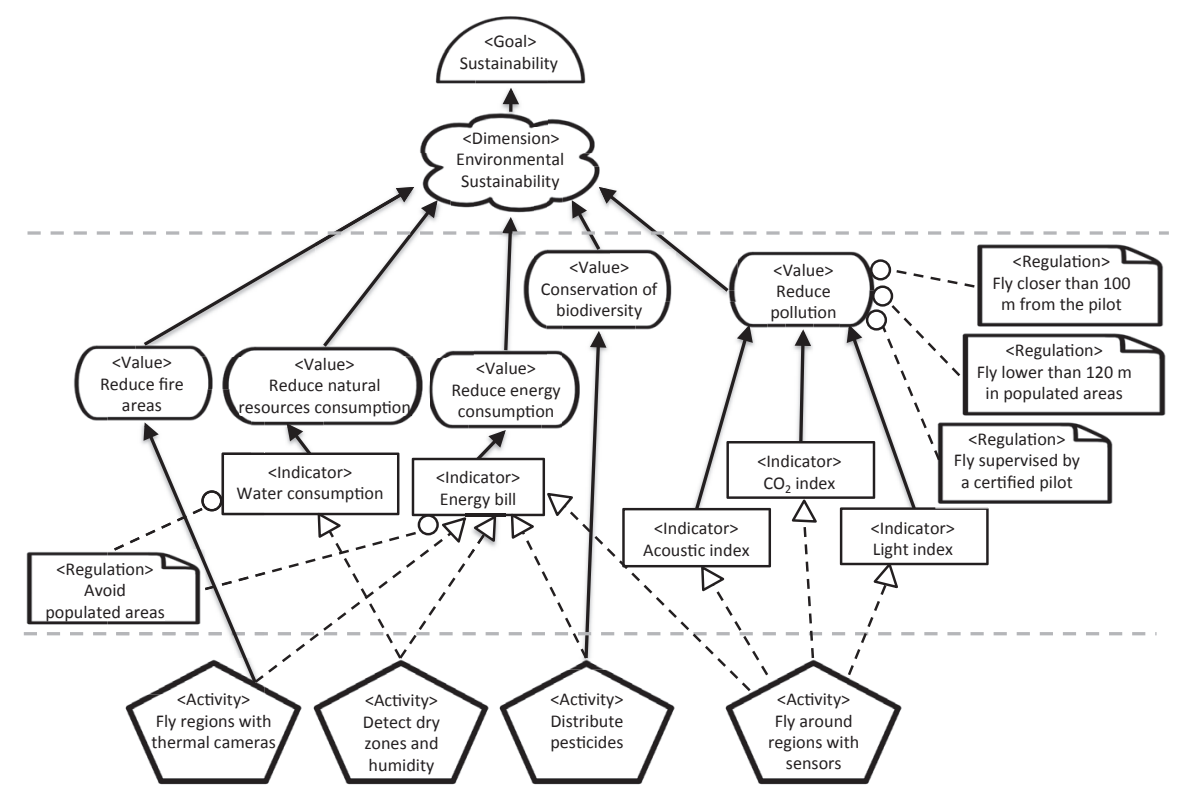

Fig. 3. Environmental dimension in the Sustainability model

financial benefits, e.g. the "Fly regions with thermal cameras" activity or the "Water bill" and "Energy bill" indicators.

As it may be observed in Fig. 4, there are several activities performed by drones that influence on some indicators that contribute to achieve financial values. As an example, the "Detect dry zones and humidity" activity influences on the "Water bill" due to the important amount of water that may be saved when irrigation is dynamically adapted. This saving in water is obviously translated into a corresponding saving in money. However, it is worth mention that the activities performed by drones also influence on some indicators that imply an increase in the costs for the organization. This is the case of "Cost of UAVs licenses and pilots" and "Cost of UAVs and maintenance" indicators. These indicators reflect new costs that the organization must afford and, thus, they help to approximate the "Evaluate cost of new processes" value. In that sense, a trade off analysis among the financial benefits and costs should be performed to evaluate the global impact of these new processes on the economy of the organization.

Observe also that the "Fly through populated areas" regulation shown in Fig. 4 also supports the indicator related to the costs of licenses and pilots since the local regulation (from Spain) establishes that a fly through a populated area must be always supervised by a licensed pilot.

\section{Technical sustainability involved in the use of UAVs}

The technical dimension of sustainability fosters technology that may be easily adapted to future changes so that its long-term use is guaranteed. Obviously, from a software engineering perspective, the achievement of this goal is clearly related to well-known quality indicators that influence this long-term use of a software product, e.g. adaptability, flexibility, maintainability or reusability. Thus, technical sustainability in the UAVs domain is also related to enhancing these quality characteristics in the software products built to control the devices and, also, in the construction of the own devices (e.g. reusing pieces or generating low cost ones). Fig. 5 shows a fragment of the model built for specifying technical sustainability for our motivating example.

It is worth remark that an important activity to ensure a long-term usage of software is the implementation of adaptive software (also claimed in [12], [27]). Observe that, in our example, the "Building adaptive software" activity influences some of the indicators shown in the model. For instance, software that may be easily adapted to changes in the aerial regulations would help to maintain technical sustainability in the organization. Even, these adaptations could be automatically applied by the own system that could be self-adapted to the environment conditions like weather changes or detection of obstacles (see sub-activities in Fig. 5).

\section{Social sustainability supported by UAVs}

The social dimension refers to all the activities that improve the values associated to a society in terms of solidarity, equality, well-being, etc. There are several domains where UAVs are being currently used that foster these social values, mainly related to medical support, security, or entertainment. However, there are also other social values that may be jeopardized by the use of these new devices. As an example, since these devices are being used, in many cases, for recording images, an obvious problem of privacy may arise from this context. Fig. 6 shows an excerpt of the model built to represent the social sustainability dimension in the context of UAVs. Note that there are four values defined: "Increase security", "Preserve privacy", "Provide 


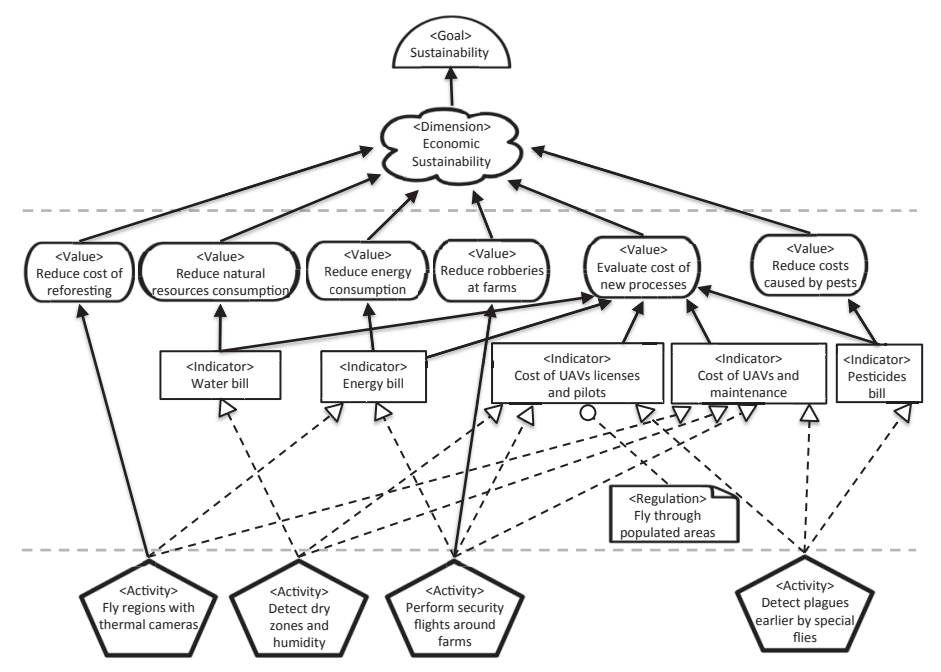

Fig. 4. Economic dimension in the Sustainability model

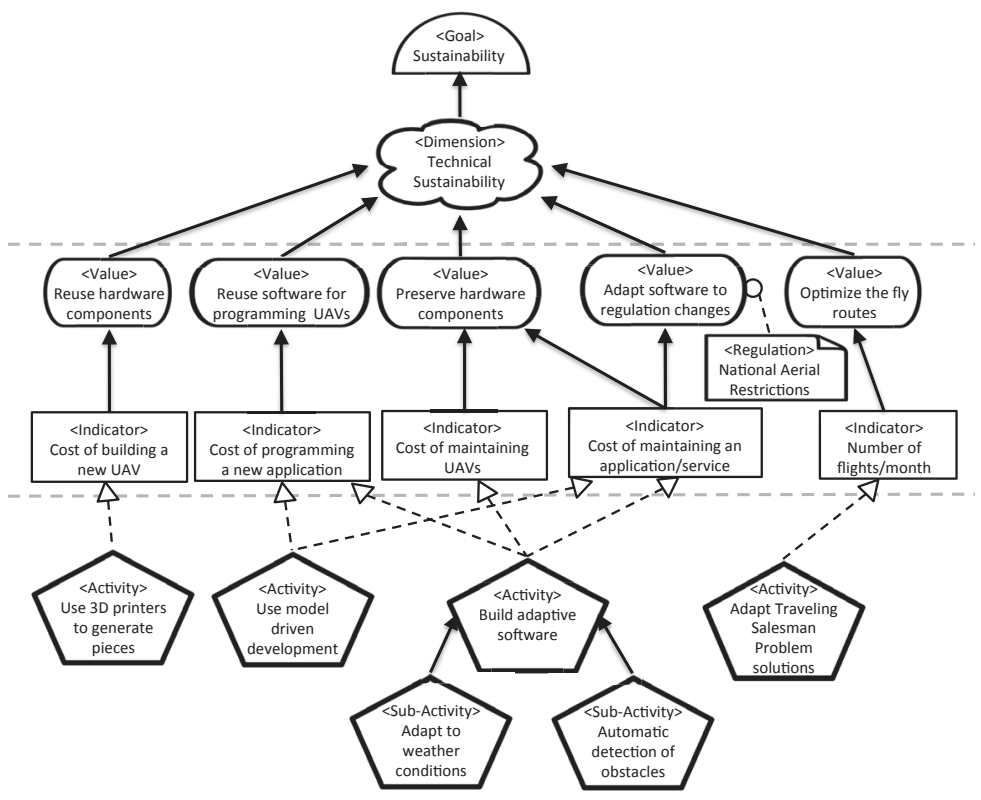

Fig. 5. Technical dimension in the Sustainability model

entertainment" and "Improve health services". Observe that while security, entertainment and health issues are clearly benefited from the use of UAVs, the relationships among the activities and the "Preserve privacy" value have a negative contribution, even, although some kind of flights may be restricted by the regulation and they may be compulsorily performed in non-populated areas.

\section{E. Lessons learnt}

Based on the experience of building the new instance of the sustainability metamodel for the UAVs domain, we observed some issues that limit the model expressivity. This section discusses these issues and provides some suggestions that could be considered to ease the process of building new instances.

1) Positive and negative contributions: although the process defined in [10] to build the models includes a step to analyze whether the activities positively or negatively influence the indicators, the relationships defined in the metamodel do not allow to express those contributions. For instance, note that this influence may be positive, e.g. if a drone helps to reduce fuel consumption, or negative, e.g. if the same drone leads to other type of energy consumption or introduces additional costs. As a different example, in the social dimension, privacy issues may be clearly jeopardized by 


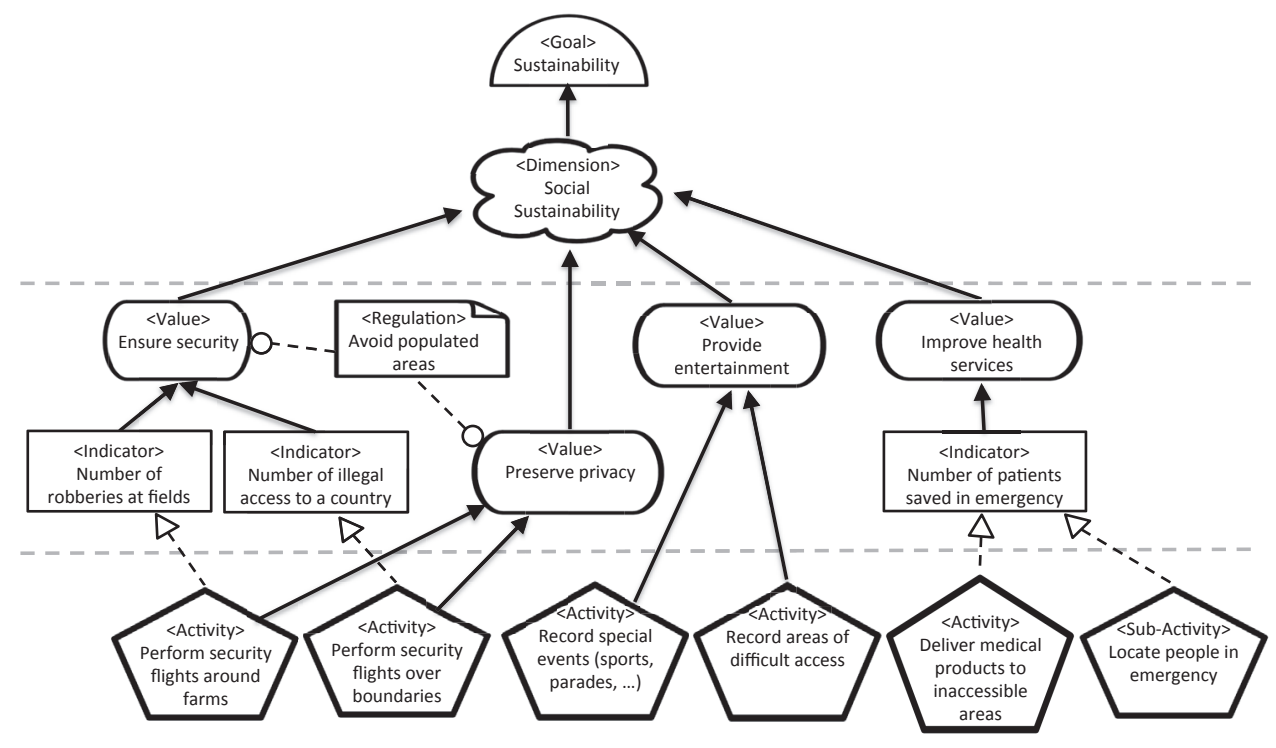

Fig. 6. Social dimension in the Sustainability model

the use of drones. In that sense, the inclusion of positive and negative influences in the metamodel as different relationships (or, at least, the inclusion of association classes) would help to clarify the models built.

2) Trade off analysis: similarly to the previous issue, we also found difficulties to measure the impact of some activities on the general goal (sustainability) when these activities enable opposite effects. For example, we claimed that the use of drones may drastically reduce the fuel consumed when these devices are used to replace traditional vehicles. However, since drones usually rely on batteries, their autonomy may be limited and, thus, the number of drones needed to cover a big area may be higher than the number of traditional vehicles. Also, the problem of privacy could hinder the utilization of drones in some contexts where other benefits could be obtained, e.g. improving security. In these cases, a trade off analysis should be performed to assess the global impact of the new processes by evaluating both the positive and negative contributions. Indeed, although positive and negative contributions were represented in the model, the information needed to perform such a trade off analysis would not be included. The use of weights or measures in the indicators could contribute to partially solve this situation.

3) Regulations: although the metamodel allows expressing how the regulations affect indicators and values, we also observed that some regulations may directly influence activities. In our case, this makes sense since aerial regulations may limit the applicability of concrete activities. Thus, the inclusion of a relationship between Regulation and Activity metaclasses would aim at representing those situations. Moreover, we also detected that a regulation not always supports a value, it may also restrict it.

4) Values: although the metamodel includes a self-relationship for the value metaclass (called "subvalue"), we also identified the need for expressing that a value "contributes" to the achievement of other values. Observe, for instance, that "Reduction of fire areas" value (in Fig. 3) also contributes to achieve other values, such as "Conservation of biodiversity" or "Reduce natural resources consumption".

\section{RELATED WORK}

Some existing works provide mechanisms and frameworks to model sustainability concepts in software engineering [28], [10], [22], [11], [29], [30], [12]. Penzenstadler and Femmer [28], [10] introduced a metamodel used to instantiate generic models for sustainability (this work was described in Section 2.2). Similarly, Naumann et al. proposed a framework to help developers implement sustainable web pages [22]. Cabot et al. provide an example of how to model sustainability goals for the organization of a conference by using $i^{*}$ models [11]. This work was extended in [29] to cope with environmental sustainability by using goal modeling techniques.

The work in [30] also proposed the use of goal oriented models but focusing on specific requirements related to time cost. Combemale et al. also highlighted the importance of models for sustainability in software development [12]. In 
particular, they claim that engineering models should be complemented with scientific ones as a way to not only build a sustainable product but also to understand the influence between the various dimensions of sustainability and balance the trade-offs among them.

Unlike all the above approaches, the work presented in this paper focuses on providing a new instantiation of a sustainability model for a concrete domain whose applications are usually related to sustainability. Note that most of these approaches (including ours) consider sustainability at a high abstraction level (modeling) and at an early stage of development (requirements). The importance of considering sustainability from the very beginning of the development was also highlighted in [13]. In that sense, the work in [27] presented a survey of the current works on requirements and sustainability by using some demonstrative examples to show how the different requirements activities may be performed from a sustainability perspective. In this work, the authors claim that while the generic sustainability metamodel described in [10] should be instantiated when sustainability is considered a major purpose of the system, the utilization of an overall goal model and a reference to a sustainability sub-model is more suitable when sustainability is treated as another objective of the system. In our case, we instantiated the metamodel since our main purpose was to evaluate the impact of new processes on sustainability, in contrast to other works that have also dealt with sustainability at the requirements level but as another non-functional requirement in the system [31], [32].

Software sustainability has been also treated from a quality perspective. Indeed, there are some works that have extended traditional quality frameworks (like ISO/IEC 25000) with new concepts related to sustainability [33] or that have analyzed the relationship between sustainability concerns and other quality requirements [34], [35], especially security [36]. In that sense, the model for sustainability presented here may help to specify the relations among drones and sustainability but also the security restrictions imposed by local regulations.

\section{CONCLUSIONS}

This paper presented a new instantiation of a generic sustainability metamodel for the context of UAVs. This instantiation allows to model different processes based on the utilization of UAVs that may be included in an organization obtaining, thus, innovative benefits with respect to traditional processes. This instantiation could be used by the managers of the organization to approximate the impact of incorporating these technologies and their degree of benefits with respect to different sustainability dimensions. Moreover, the model not only allows modeling those benefits but also specifying the limitations, for instance, due to strict aerial regulations. Finally, some suggestions to complete the metamodel were also provided.

In the near future, we plan to extend the metamodel introduced in [10] to address the issues identified (e.g. specifying conflicts or positive and negative relationships) for the UAVs domain and incorporate new concepts related to adaptability. Similarly, we plan to provide a reusable catalogue of sustainability concepts supported by drones. Finally, our plan is to provide an approach and supporting tool to identify interactions and conflicts to manage the delicate balance among the various sustainability dimensions.

\section{ACKNOWLEDGMENT}

This work has been supported by Junta de Extremadura (according to the Order 129/2015 of the 2nd of June) and NOVA LINCS Research Laboratory (Ref. UID/CEC/04516/2013).

\section{REFERENCES}

[1] P. Tokekar, J. Vander Hook, D. Mulla, and V. Isler, "Sensor planning for a symbiotic UAV and UGV system for precision agriculture," in 2013 IEEE/RSJ International Conference on Intelligent Robots and Systems. IEEE, nov 2013, pp. 5321-5326. [Online]. Available: http: //ieeexplore.ieee.org/lpdocs/epic03/wrapper.htm?arnumber $=6697126$

[2] P. B. Quater, F. Grimaccia, S. Leva, M. Mussetta, and M. Aghaei, "Light Unmanned Aerial Vehicles (UAVs) for Cooperative Inspection of PV Plants," IEEE Journal of Photovoltaics, vol. 4, no. 4, pp. 1107-1113, jul 2014. [Online]. Available: http://ieeexplore.ieee.org/ lpdocs/epic03/wrapper.htm?arnumber=6828739

[3] L. Zhang, B. Wang, W. Peng, C. Li, Z. Lu, and Y. Guo, "A Method for Forest Fire Detection Using UAV."

[4] L. Merino, F. Caballero, J. R. Martínez-De-Dios, I. Maza, and A. Ollero, "An Unmanned Aircraft System for Automatic Forest Fire Monitoring and Measurement," Journal of Intelligent \& Robotic Systems manuscript, vol. 65, no. 1, pp. 533-548, 2012.

[5] C. Yuan, Z. Liu, and Y. Zhang, "UAV-based forest fire detection and tracking using image processing techniques," in 2015 International Conference on Unmanned Aircraft Systems (ICUAS). IEEE, jun 2015, pp. 639-643. [Online]. Available: http://ieeexplore.ieee.org/ lpdocs/epic03/wrapper.htm?arnumber=7152345

[6] P. Sujit, J. Sousa, and F. L. Pereira, "UAV and AUVs coordination for ocean exploration," in OCEANS 2009-EUROPE. IEEE, may 2009, pp. 1-7. [Online]. Available: http://ieeexplore.ieee.org/lpdocs/epic03/ wrapper.htm?arnumber $=5278262$

[7] I. Maza, F. Caballero, J. Capitán, J. R. Martínez-de Dios, and A. Ollero, "Experimental Results in Multi-UAV Coordination for Disaster Management and Civil Security Applications," Journal of Intelligent \& Robotic Systems, vol. 61, no. 1-4, pp. 563-585, jan 2011. [Online]. Available: http://link.springer.com/10.1007/s10846-010-9497-5

[8] A. M. Samad, N. Kamarulzaman, M. A. Hamdani, T. A. Mastor, and K. A. Hashim, "The potential of Unmanned Aerial Vehicle (UAV) for civilian and mapping application," in 2013 IEEE 3rd International Conference on System Engineering and Technology. IEEE, aug 2013, pp. 313-318. [Online]. Available: http://ieeexplore. ieee.org/lpdocs/epic03/wrapper.htm?arnumber=6650191

[9] H. Blair-Smith, "Aviation mandates in an automated fossil-free century," in 2015 IEEE/AIAA 34th Digital Avionics Systems Conference (DASC). IEEE, sep 2015, pp. 9A3-1-9A3-9. [Online]. Available: http: //ieeexplore.ieee.org/lpdocs/epic03/wrapper.htm?arnumber $=7311485$

[10] B. Penzenstadler and H. Femmer, "A generic model for sustainability with process- and product-specific instances," in Workshop on Green in/by software engineering - GIBSE. New York, New York, USA: ACM Press, 2013, p. 3. [Online]. Available: http: $/ /$ dl.acm.org/citation.cfm?doid=2451605.2451609

[11] J. Cabot, S. Easterbrook, J. Horkoff, L. Lessard, S. Liaskos, and J.-N. Mazon, "Integrating sustainability in decision-making processes: A modelling strategy," in 31st International Conference on Software Engineering. IEEE, 2009, pp. 207-210. [Online]. Available: http: //ieeexplore.ieee.org/lpdocs/epic03/wrapper.htm?arnumber=5070983

[12] B. Combemale, B. H. C. Cheng, A. Moreira, and J. Gray, "Modeling for Sustainability," in ACM Modeling in Software Engineering (MISE), 2016, pp. 62-66.

[13] C. Becker, S. Betz, R. Chitchyan, L. Duboc, S. M. Easterbrook, N. Seyff, and C. C. Venters, "Requirements : The Key to Sustainability," IEEE Software, vol. 33, no. 1, pp. 56-65, 2016. 
[14] R. Pereira, M. Couto, J. Saraiva, J. Cunha, and J. P. Fernandes, "The influence of the Java collection framework on overall energy consumption," in Proceedings of the 5th International Workshop on Green and Sustainable Software - GREENS '16. New York, New York, USA: ACM Press, 2016, pp. 15-21. [Online]. Available: http://dl.acm.org/citation.cfm?doid=2896967.2896968

[15] M. Couto, J. Cunha, J. P. Fernandes, R. Pereira, and J. Saraiva, "GreenDroid: A tool for analysing power consumption in the android ecosystem," in 2015 IEEE 13th International Scientific Conference on Informatics. IEEE, nov 2015, pp. 73-78. [Online]. Available: http: //ieeexplore.ieee.org/lpdocs/epic03/wrapper.htm?arnumber=7377811

[16] M. Couto, T. Carção, J. Cunha, J. P. Fernandes, and J. Saraiva, "Detecting Anomalous Energy Consumption in Android Applications," in Proceedings of the 18th Brazilian Symposium on Programming Languages. Springer International Publishing, 2014, pp. 77-91. [Online]. Available: http://link.springer.com/10.1007/978-3-319-11863-5_6

[17] P. G. Neumann, "The foresight saga," Communications of the $A C M$, vol. 55, no. 10, pp. 26-29, 2012. [Online]. Available: http://portal.acm.org/citation.cfm?doid=1151030.1151060

[18] C. Becker, R. Chitchyan, L. Duboc, S. Easterbrook, B. Penzenstadler, N. Seyff, and C. C. Venters, "Sustainability Design and Software: The Karlskrona Manifesto," Proceedings - International Conference on Software Engineering, vol. 2, no. May, pp. 467-476, 2015.

[19] P. Lago, "Challenges and opportunities for sustainable software," in Proceedings of the Fifth International Workshop on Product LinE Approaches in Software Engineering. IEEE Press, 2015, pp. 1-2.

[20] B. Penzenstadler, A. Raturi, D. Richardson, C. Calero, H. Femmer, and X. Franch, "Systematic mapping study on software engineering for sustainability (SE4S)," in Proceedings of the 18th International Conference on Evaluation and Assessment in Software Engineering - EASE '14. New York, New York, USA: ACM Press, 2014, pp. 1-14. [Online]. Available: http://dl.acm.org/citation.cfm?doid=2601248. 2601256

[21] C. Venters, C. Jay, L. Lau, M. Griffiths, V. Holmes, R. Ward, J. Austin, C. Dibsdale, and J. Xu, "Software Sustainability: The Modern Tower of Babel," in International Workshop on Requirements Engineering for Sustainable Systems. CEUR, 2014, pp. 7-12.

[22] S. Naumann, M. Dick, E. Kern, and T. Johann, "The GREENSOFT Model: A reference model for green and sustainable software and its engineering," Sustainable Computing: Informatics and Systems, vol. 1, no. 4, pp. 294-304, 2011

[23] UN World Comission on Environment and Development, "Our Common Future: Report of the World Commission on Environment and Development," Tech. Rep.
[24] R. Goodland, "Sustainability : Human , Social , Economic and Environmental," in Encyclopedia of Global Environmental Change, W. \&. Sons, Ed., 2002, vol. 6, no. 11, pp. 220-225. [Online] Available: www.balticuniv.uu.se/index.php/download/doc_download/ 435-sustainability-human-social-economic-and-environmental

[25] UK UAVs Association, "UAV or UAS?" [Online]. Available: https://www.uavs.org/index.php?page=what_is

[26] M. Johnson, "Components for Creating an Unmanned Aerial Vehicle," Tech. Rep. [Online]. Available: http://www.egr.msu.edu/classes/ece480/capstone/spring15/ group14/uploads/4/2/0/3/42036453/johnsonappnote.pdf

[27] R. Chitchyan, S. Betz, L. Duboc, B. Penzenstadler, C. Ponsard, and C. C. Venters, "Evidencing sustainability design through examples," in RE4SuSy Workshop at Requirements Engineering Conference, vol. 1416, 2015, pp. 45-54.

[28] B. Penzenstadler and H. Femmer, "A Generic Model for Sustainability," Tech. Rep., 2012.

[29] D. Stefan and E. Letier, "Goal-Oriented System Modelling for Managing Environmental Sustainability," in WSRCC, 2010.

[30] G. Mussbacher and D. Nuttall, "Goal modeling for sustainability: The case of time," in 4th International Model-Driven Requirements Engineering Workshop, MoDRE, Karlskrona, Sweden, 2014, pp. 7-16.

[31] C. C. Venters, L. Lau, M. K. Griffiths, V. Holmes, R. R. Ward, C. Jay, C. E. Dibsdale, and J. Xu, "The Blind Men and the Elephant Towards an Empirical Evaluation Framework for Software Sustainability," Jorunal of Open Research Software, vol. 2, no. 1, pp. 1-6, 2014

[32] B. Penzenstadler, A. Raturi, D. Richardson, and B. Tomlinson, "Safety, Security, Now Sustainability: The Nonfunctional Requirement for the 21st Century," IEEE Software, vol. 31, no. 3, pp. 40-47, may 2014. [Online]. Available: http://ieeexplore.ieee.org/lpdocs/epic03/ wrapper.htm?arnumber $=6728940$

[33] C. Calero, M. angeles Moraga, M. F. Bertoa, and L. Duboc, "Quality in use and software greenability," in RE4SuSy Workshop at Requirements Engineering Conference, vol. 1216, 2014, pp. 28-36. [Online]. Available: http://ceur-ws.org/Vol-1216/paper6.pdf

[34] N. C. Fernandez, P. Lago, and C. Calero, "How do Quality Requirements Contribute to Software Sustainability?" in REFSQ Workshop, 2016, pp. 7-10. [Online]. Available: http://ceur-ws.org/Vol-1564/paper33.pdf

[35] S. A. Koçak, G. I. Alptekin, and A. B. Bener, "Integrating Environmental Sustainability in Software Product Quality," 2015.

[36] G. Rodriguez-Navas, L. Duboc, S. Betz, R. Chitchyan, B. Penzenstadler, and C. C. Venters, "Safety vs. sustainability design: Analogies, differences and potential synergies," CEUR Workshop Proceedings, vol. 1416, pp. 25-34, 2015. 